\title{
Review of: "Home alone: A population neuroscience investigation of brain morphology substrates"
}

\author{
Fatemeh Darabi
}

Potential competing interests: The author(s) declared that no potential competing interests exist.

Review of: "Home alone: A population neuroscience investigation of brain morphology substrates"

This is study in a in the field of neuroscience.

There are a number of concerns with this study which include the following:

- Most of the references used are outdated and need to be updated.

- In the abstract section, the statistical results should be mentioned only descriptively and very briefly.

- Incomplete results are written in the abstract.

- Write the final research proposal in the discussion.

- Match the keywords with the mesh.

- The importance of the subject and the reason for the study is very incomplete and should be completed.

- Explain how to conduct the study and how to observe ethical issues.

- Mention the study method and entry and exit criteria.

- Explain how to sample in the introduction.

- In the discussion section, first write the main results of the study and then compare it with other studies.

- Give your reasons.

- Complete the conclusion.

- Make suggestions.

- Write the strengths and weaknesses of the study. 